\title{
What are the functions of nails?
}

\author{
Inderbir Padda ${ }^{1}$ \\ 1 University of Washington
}

Funding: The author(s) received no specific funding for this work.

Potential competing interests: The author(s) declared that no potential competing interests exist.

\begin{abstract}
What are the functions of nails?

The nails embryonic origin starts composing at the seventh to eighth weeks during development and is entirely constructed at childbirth. The human anatomy of the nail comprises epithelial and connective tissue components; nail matrix, nail bed, nail plate, nail folds, mantle, hyponychium, and isthmus. The nail functions to provide sensory, thermoregulatory, and protective features to the distal fingers and toes. The average nail lengthens two-three millimeters every four weeks, replacing itself entirely once every six-nine months, depending on the individual. The nail growth, color, consistency, and appearance may provide insight into an individual's health and manifestations of localized or deeper systemic conditions
\end{abstract}

What does the following nail conditions reveal about the overall health?

\section{Yellowing of nails}

One of the leading explanations of yellow discoloration of the nails can be a fungal infection of the nail folds and subungual tissue, also known as "Onychomycosis." This can oftentimes be characterized by flaking of the nails leading to nail thickening and crumbling, accompanied by an unpleasant odor. [1] Depending on the severity of the infection, it can be localized to one digit or involve all five nails bilaterally. Although more commonly affecting the toes, it may also affect the fingers. An infrequent and rare cause of yellowing of the nail is "yellow nail syndrome." This condition's etiology is still unclear but is said to be associated with a dysfunction of the lymphatic drainage. This is often confused with Onychomycosis as the nails may become thick and hard, presenting very similarly. ${ }^{[2]}$

\section{Pale and dull nails}

Nail discoloration is one of the signs of advances in aging. ${ }^{[3]}$ As humans age, the nail morphology can tend to take a duller, more pale, and whitish appearance. ${ }^{[3]}$ Although physiologic in the senile age, its presence in early age in life may be idiopathic or indicative of underlying disease. Whitish discoloration of the nails, also known as "leukonychia," can present similar to pale nails. Muehrcke's nail or Muehrcke's lines are a form of leukonychia, when present, may be indicative of an underlying systemic disease. These nails present a whitish discoloration that can fade when applying 
pressure to the surface of the nail plate. This is predominantly a manifestation of "hypoalbuminemia" which is a term for decreased blood albumin levels. ${ }^{[4]}$ Albumin levels can be low from reduced production due to liver disease or increased excretion from the kidneys from renal disease, mainly nephrotic syndrome. Other conditions that may cause hypoalbuminemia are systemic inflammatory disease and individuals receiving chemotherapy. ${ }^{[4]}$

\section{Cracked and split nails}

Nail brittleness can result in cracked and split nails. The two common types of presentation for brittle nails are "Onychoschizia" and "Onychorrhexis."[5] Onychoschizia is a term for the horizontal distal splitting of the nail's free end and can also be referred to as lamellar splitting. This occurs most commonly from trauma, causing separation to the adhesions of the nail plate intracellularly. ${ }^{[5]}$ It can also occur from frequent washing of the hands. Onychorrhexis, similarly to Onychoschizia, present as longitudinal splitting rather than horizontal splitting. This can present either as a single split or several longitudinal splits of the superficial nail plate. This can occur as an idiopathic occurrence with no underlying identifiable cause or can be due to various conditions such as trauma, nail-biting, alopecia areata, eczema, psoriasis, anemia, or bulimia nervosa. ${ }^{[5]}$ Nutritional deficiencies can also have an effect on the development of nail splitting.

\section{Excessively thick nails}

A few causes of nail thickening may be due to fungal infection "Onychomycosis," as discussed earlier, and psoriasis of the nail. These conditions cause excessive keratinocytes to proliferate at the nail bed's distal end and debris to build up towards the proximal end of the nail, also known as "subungual hyperkeratosis."[6] Psoriasis of the nail is known to occur as a dysregulated immunological response of the innate immunity. A genetic component has been associated but not confirmed. The presentations can be similar between both onychomycosis and psoriasis of the nail and should be investigated thoroughly, as misdiagnosis may lead to delays in management and outcome.

\section{Clubbing of the nails}

Clubbing of the nails refers to the convex appearance of the nail. The standard anatomical angle of a healthy nail is 160 degrees and is considered "clubbing" when the angle between proximal nail fold and the nail plate exceeds 180 degrees. [7] This type of nail disorder can be secondary to acquired etiologies such as vascular, cancer, inflammatory, and infection.

[7] It may also occur solely without underlying pathologies as a benign inheritance. Decreased blood oxygen levels, also referred to as "hypoxemia," secondary to pulmonary pathology (malignancy, interstitial lung disease, cystic fibrosis) is one of the predominant causes of nail clubbing.

\section{Spoon nails}

Spoon nails, also known as "Koilonychia," is termed when the nails have a concave appearance that can be either horizontal or vertical. ${ }^{[8]}$ These nails are characterized as brittle and thin in either the fingernails or toenails; spoon's nails can be seen in all age groups. This condition can be hereditary, congenital, acquired, or idiopathic. ${ }^{\left[{ }^{8]}\right.}$ When spoons nails are present, it is vital to rule out an underlying systemic cause that may be occurring. Spoon nails most frequently develop as a manifestation of severe chronic iron deficiency anemia. The origins of iron deficiency are aplenty and should be 
investigated thoroughly for systemic or chronic diseases that may be occurring.

\section{Pitted nails}

Nail pitting, also termed "trachyonychia," presents as studding or denoting dots or depressions on the nail plate's surface. ${ }^{[9]}$ This occurs due to defective keratinization of the proximal nail matrix and surface. ${ }^{[9]}$ The presence of nail pitting may be idiopathic or can be a manifestation of conditions such as psoriasis, alopecia areata, eczema, and chronic renal failure. Other uncommon causes include SLE, sarcoidosis, dermatomyositis, and syphilis. ${ }^{[9]}$

\section{Terry's nails}

Terry's nails may present as a whitish discoloration, also known as "leukonychia," with a pinkish brown stripe at the tip. It can also be characterized as 0.5 - to - 3.0 - mm wide, brown to pink nail bed bands. ${ }^{[10]}$ Terry's nails are predominantly seen in the fingernails, but their presence in toenails is not uncommon. All fingernails are commonly affected equally, but it may also present solely on one digit. When this is present, it may be suggestive of an underlying systemic disease. ${ }^{[11]}$ This includes liver disease, chronic kidney failure, congestive heart failure, HIV, diabetes, and peripheral vascular disease. ${ }^{[11]}$ Terry's nails are not to be confused with Muehrcke's nails as both may present as a whitish discoloration but should be differentiated as both presents from different underlying systemic disorders.

\section{Horizontal depressions}

Horizontal or transverse depression in the nail is referred to as "Beau's lines." Beau's lines extend the whole width of the nail with a band-like depression. ${ }^{[9]}$ The etiology for this manifestation is due to a growth cessation of the nail matrix. ${ }^{[9]}$ This is thought to be a temporary cessation that may be precipitated by inadequate nutritional health, acute events such as a heart attack, trauma, infection, and stress. A full termination in growth will result in "onychomadesis," which is a group of band-like depressions. ${ }^{[9]}$ Its presence in all digits of hands and feet is indicative of a systemic disease that may be occurring. This includes Kawasaki disease, mumps, pulmonary, and cardiovascular pathology. [12]

\section{What do pale nails signify?}

Pale nails can signify aging, anemia, or more serious illness like liver disease.

\section{Is horizontal depression a common phenomenon seen in nails?}

Horizontal depressions are also known as "beaus lines." They are not very common in healthy individuals and may signify an underlying health condition.

\section{Can nail fungus and psoriasis cause cracked and split nails?}

Yes, nail fungus and psoriasis can cause cracked and split nails.

\section{Can signs of cardiovascular diseases be shown in nails?}

Yes, signs of cardiovascular disease can be shown in nails. It may present as nail clubbing or bluish tint in nails which can 
be from decreased oxygen levels in the blood. Endocarditis, a bacterial infection that affects the heart valve, can cause splinter hemorrhages in the nail, which can present linear reddish-brown streaks in the nail.

Which nutrient deficiency can cause which nail symptoms?

Iron, Zinc, Biotin(B7), Folate (B9), and Cobalamin(B12) can all cause nail symptoms. Iron deficiency can cause a pale appearance to the nails and a severe iron deficiency can cause anemia and may further develop spoon nails(koilonychia). Zinc deficiency can cause white spots to develops on the nail plate. Cobalamin(B12) deficiency can cause dark nail discoloration. Folate (B9) deficiency can cause pigmentation and brittle nails. Biotin(B7) can cause brittle nails.

\section{What do white spots on the nails signify?}

Whitish discoloration of the nails, also known as "leukonychia," can be idiopathic, signs of aging, or can occur from anemia, malnutrition, trauma, or stress-induced. Terry's nails and Muehrcke's lines are variations of leukonychia and may be a manifestation of an underlying disease affecting the liver or kidneys.

\section{What do healthy nails look like?}

Healthy nails are smooth, durable, and even in texture; the nail bed is pink, whereas the nail margin is white without ridges. Healthy nails should not be brittle, excessively thick, have depressions, splits, streaks, or discoloration. To maintain the appearance of healthy nails, it is beneficial to practice grooming methods such as nail cutting and aesthetic products to strengthen nails. Along with sensory and protective functions, the cosmetic feature of nails is equally significant.

\section{What dietary changes should be made to strengthen the nails?}

A nutrient-dense diet that is low in sugar, sodium, and saturated fats can strengthen nails alongside hair and skin. Nutritional dietary supplements such as B vitamins; biotin(B7), folate(B9), cobalamin(b12), and minerals; iron and zinc can also be incorporated.

\section{Is it normal for the fingernails to be yellow when one grows old?}

A yellowish gray discoloration may be observed, but it is uncommon for fingernails to be yellow in the elderly. However, it is not uncommon for nails to be dull or pale as a person ages. Chronic smokers may also display yellowing of the fingernails caused by staining from nicotine.

What do dark lines on the fingernails signify?

As mentioned earlier, endocarditis may cause splinter hemorrhages in the nail, which can present linear reddish-brown streaks in the nail. It can also be the beginning stages of a fungal infection, or may also signify a more serious condition such as melanoma. 
nail morphologies can be idiopathic, acquired, hereditary, or a manifestation of underlying health concerns.

\section{References}

1. ^ Johnson C, Sinkler MA, Schmieder GJ. (2020). Anatomy, Shoulder and Upper Limb, Nails.. Statpearls.

2. ^ Cheslock M, Harrington Dw.. (2020). Yellow Nail Syndrome. StatPearls Publishing.

3. ${ }^{a, b}$ Ramachandran V, Sapra A.. (2020). Muehrcke Lines Of The Fingernails. StatPearls Publishing.

4. ${ }^{a, b}$ Abdullah L, Abbas O.. (2011). Common nail changes and disorders in older people: Diagnosis and management. Canadian family physician Medecin de famille canadien, vol. vol. 57,2 (2011): 173-81. . Can Fam Physician.

5. a, b, c Marco A. Chessa, Matilde lorizzo, Bertrand Richert, Jose L. López-Estebaranz, Dimitrios Rigopoulos, Antonella Tosti. (2019). Pathogenesis, Clinical Signs and Treatment Recommendations in Brittle Nails: A Review. Dermatol Ther (Heidelb), vol. 10 (1), 15-27. doi:10.1007/s13555-019-00338-x.

6. ^ Muneer H, Masood S. (2020). Psoriasis of the Nails. Statpearls Publishing.

7. ${ }^{a, b}$ Burcovschii S, Aboeed A. (2020). Nail Clubbing. Statpearls Publishing.

8. ${ }^{\text {a, b }}$ Rathod DG, Sonthalia S. (2020). Spoon Nails (Koilonychia). Statpearls Publishing.

9. a, b, c, d, e, f Archana Singal, Rahul Arora. (2015). Nail as a window of systemic diseases. Indian Dermatol Online J, vol. 6 (2), 67. doi:10.4103/2229-5178.153002.

10. ^ Cashman MW, Sloan SB. (2010). Nutrition and nail disease. Clinics in dermatology, vol. vol. 28,4 (2010): $420-5$.

11. ${ }^{a, b}$ Witkowska AB, Jasterzbski TJ, Schwartz RA. (2017). Terry's Nails: A Sign of Systemic Disease.. Indian journal of dermatology, vol. vol. 62,3 (2017): 309-311.

12. ^ Eckart Haneke. (2015). Anatomy of the nail unit and the nail biopsy. Sem Cutan Med Surg, vol. 34 (2), 95-100. doi:10.12788/j.sder.2015.0143. 